\title{
1 The projected timing of abrupt ecological disruption from climate
}

2 change

3

4 Authors: Christopher H. Trisos ${ }^{1,2,3}$, Cory Merow ${ }^{4}$, Alex L. Pigot ${ }^{5 *}$

5 Affiliations: ${ }^{1}$ African Climate and Development Initiative, University of Cape Town, South

6 Africa; ${ }^{2}$ National Socio-Environmental Synthesis Center (SESYNC), 1 Park Place, Suite 300,

7 Annapolis, MD 21401, USA; ${ }^{3}$ Centre for Statistics in Ecology, the Environment, and

8 Conservation, University of Cape Town, South Africa; ${ }^{4}$ Ecology and Evolutionary Biology,

9 University of Connecticut, Storrs, CT 06269, USA; ${ }^{5}$ Centre for Biodiversity and Environment

10 Research, Department of Genetics, Evolution and Environment, University College London,

11 Gower Street, London, WC1E 6BT, UK.

12 *Correspondence to: a.pigot@ucl.ac.uk 
13 As anthropogenic climate change continues, the risks to biodiversity will increase 14 over time, with future projections indicating a potentially catastrophic loss of global 15 biodiversity on the horizon ${ }^{1-3}$. However, our understanding of how this climate16 driven disruption of biodiversity will unfold over time is limited because biodiversity forecasts typically focus on individual snapshots of the future. Here, we use annual projections (1850-2100) of temperature and precipitation across $>30,000$ marine and terrestrial species' ranges to estimate the timing of species exposure to potentially dangerous climate conditions. We project that future disruption of ecological assemblages from climate change will be abrupt, because within any given ecological assemblage the exposure of most species to climate conditions beyond their realised niche limits occurs near simultaneously. Under a high emissions scenario (RCP8.5), such abrupt exposure events begin before 2030 in tropical oceans and spread to tropical forests and higher latitudes by 2050 . Below $2{ }^{\circ} \mathrm{C}$ global warming, $<2 \%$ of assemblages globally are projected to undergo abrupt exposure events of $>20 \%$ of their constituent species, but the risk accelerates with the magnitude of warming, threatening $15 \%$ of assemblages at $4^{\circ} \mathrm{C}$, with similar levels of risk in protected and unprotected areas. These results highlight the impending risk of sudden and severe biodiversity losses from climate change and provide a framework for predicting both when and where these events may occur. 


\section{Introduction}

Climate change is projected to become a leading driver of biodiversity loss ${ }^{1}$, but when during this century might ecological assemblages suffer such losses, and will the process be gradual or abrupt? Existing biodiversity forecasts typically lack the temporal perspective needed to answer this question because they indicate the number and locations of species threatened by climate change for just a snapshot of the future, often around the end of the century ${ }^{1-3}$. These snapshots do not account for the temporally dynamic nature of ecological disruption expected due to climate change, often focus at the level of species rather than ecological assemblages, and can seem remote to decision-makers concerned with managing more immediate risks 4 . Indeed, many of the most sudden and severe ecological impacts of climate change can occur when conditions become unsuitable for multiple co-occurring species simultaneously, causing catastrophic die-offs and abrupt 'regime shifts' in ecological assemblages ${ }^{5,6}$.

Forecasting the temporal dynamics of climate-driven disruption of ecological assemblages thus requires quantifying the differences among species in the time at which their climate niche limits may be locally exceeded. Developing advance warnings of the risk of gradual or abrupt ecological disruption is an urgent priority ${ }^{7-9}$. A temporal perspective is also important for adaptation. Reducing emissions and delaying the onset of exposure to dangerous climate conditions, even by a few decades, could buy valuable time for ecological assemblages to adapt ${ }^{10,11}$, potentially reducing the magnitude of ecological disruption. However, despite the clear importance of a temporal perspective in understanding and managing climate change threats to biodiversity, we lack a general understanding of the time at which species in ecological assemblages will be exposed to climate conditions beyond their niche limits.

\section{The biodiversity climate horizon}

To describe the projected timing of exposure of species to climate conditions beyond their niche, we developed an approach based on species historical climate limits and future climate projections. The range of climate conditions, both over space and time, under which a species has been recorded in the wild demarcates the boundaries of its realised niche ${ }^{12}$. The projected time in the future at which these bounds are exceeded due to climate change at a site can therefore be thought of as representing a climate horizon, beyond which evidence for the species' ability to persist in the wild is lacking. Over this horizon lies, at best, a sizeable increase in uncertainty about species survival and, at worst, local extinction ${ }^{13}$. For a given species assemblage, the cumulative percentage of species over time that have been locally exposed to climate conditions exceeding their realised niche limits reveals what we term the 'horizon profile' (Fig. 1). The shape of this horizon profile provides information on the potential for climate-driven disruption of species assemblages over time-especially the risk of early or abrupt disruption — that is not evident when focusing on individual climate snapshots.

We constructed horizon profiles for species assemblages globally, delimiting assemblages as the species occurring in $100 \mathrm{~km}$ grid cells based on expert verified geographic range maps for 30,652 species of birds, mammals, reptiles, amphibians, marine fish, benthic marine invertebrates, krill, cephalopods and habitat forming corals and seagrasses ${ }^{14}$ (Supplementary Table 1 ). We used climate projections throughout the $21^{\text {st }}$ century from 22 climate models and three Representative Concentration Pathways (RCPs): 
strong mitigation (RCP2.6), moderate mitigation (RCP4.5), and a high emissions scenario (RCP8.5) ${ }^{15}$ (Supplementary Table 2). Given the importance of temperature as a driver of species metabolism and geographic ranges ${ }^{16-18}$, we focus on mean annual temperature as the main proxy for climate. However, because species may be sensitive to other climate variables that may respond differently to greenhouse gas emissions, we also generated horizon profiles using maximum monthly temperatures and terrestrial annual precipitation (see Methods).

For each species at a site (i.e., $100 \mathrm{~km}$ grid cell), we defined the local species exposure time as the year after which projected local temperatures consistently exceed, for at least five years, the maximum temperature experienced by the species across its geographic range during historical climate projections (1850-2005) (Supplementary Fig. 1). For species that breed annually or near-annually, five years represents a significant number of breeding seasons at temperatures beyond which they have never been recorded (a 20-year window yielded very similar results (Supplementary Figs. 2 and 3)). This approach for quantifying exposure bears similarities to the concept of 'time of emergence' in climate science, defined as the time at which the signal of anthropogenic climate change at a location emerges from the envelope of historical climate variability ${ }^{19,20}$. The key distinction is that we define exposure relative to each species' realised climatic niche limits rather than the historical conditions realised at a single site.

The shape of horizon profiles, and the potential ecological disruption they imply, can vary substantially across assemblages (Fig. 1). To summarize each horizon profile, we focus on three key features: (1) timing, the median year for an assemblage when species exposure to unprecedented climate occurs; (2) magnitude, the percentage of species locally exposed; and (3) abruptness, the synchronicity in the timing of exposure among species in an assemblage, measured as the percentage of all species exposure times that occur in the decade of maximum exposure (Fig. 1a).

\section{Timing, magnitude and abruptness of horizon profiles}

Under RCP8.5, 81\% of terrestrial and 37\% of marine assemblages are projected to have at least one species exposed to unprecedented mean annual temperatures (i.e., beyond historical niche limits) before 2100. Despite the lower magnitude of warming, the magnitude of exposure is greatest in the tropics, where narrow historical climate variability ${ }^{20}$ and shallow thermal gradients ${ }^{21}$, result in many species occurring near their upper realised thermal limits throughout their geographic range. In total, $68 \%$ of terrestrial and $39 \%$ of tropical marine assemblages are projected to have $>20 \%$ of their constituent species exposed to unprecedented temperatures by 2100 , compared to $7 \%$ of terrestrial and $1 \%$ of marine assemblages outside the tropics (Fig. 2a). The Amazon, Indian subcontinent and Indo-Pacific regions are most at risk, with $>90 \%$ of species in any assemblage exposed to unprecedented temperatures by 2100 (Fig. 2a). Horizon profiles for mean annual and maximum monthly temperatures show strong correspondence (Extended Data Figs. 1 and 2). In contrast, few species undergo prolonged exposure to unprecedented high or low annual precipitation before 2100 (Extended Data Figs. 1 and 2), in agreement with greater variability in precipitation projections ${ }^{22}$. Thus, throughout we focus on exposure to changes in temperature.

The most striking feature of horizon profiles for local assemblages is their abruptness (Figs. 1 and 2b). Under RCP8.5, on average 71\% (median) of local species 
exposure times for any given assemblage are projected to occur within a single decade (Fig. $3 a-b)$, with the abruptness of exposure higher among marine assemblages (median abruptness $=89 \%$, Fig. $3 \mathrm{a}$ ) than on land (median abruptness $=61 \%$., Fig. $3 \mathrm{~b}$ ). This pattern of highly synchronized species exposure within assemblages is robust to the choice of climate model (RCP8.5 median abruptness ranges from 60\% to 79\%, Extended Data Figs. 3 and 4), emissions scenario (median abruptness $=83 \%$ for RCP 2.6 and $72 \%$ for RCP 4.5 ), metric of abruptness (Extended Data Fig. 4), and when calculating exposure for maximum monthly (median abruptness $=68 \%$ ) rather than mean annual temperatures (Extended Data Figs. 1 and 2). The same pattern of abruptness is also evident for horizon profiles constructed separately for each taxonomic group within local assemblages (Extended Data Fig. 4). Marine organisms, especially seagrasses, corals, cephalopods, marine reptiles and mammals exhibit the most abrupt profiles, but it is the consistency of abruptness across groups rather than the differences that is most notable. Similarly, although abruptness for assemblages varies spatially, being greatest in the Amazon, Indian subcontinent, Sahel and Northern Australia, as well as tropical oceans, abrupt horizon profiles are the general rule both within the tropics (median abruptness $=79 \%$ ) and at higher latitudes (median abruptness $=59 \%$ ) (Fig. 2b).

This pervasive pattern of abrupt exposure arises primarily because co-occurring species often share similar realised thermal limits, rather than abruptness being dependent on higher rates of warming (Extended Data Fig. 5). Clustering of species realised thermal limits can, in part, be explained by shared geographic boundaries or, for tropical species, by the upper limits of temperatures available on Earth ${ }^{13,23}$. However, even for assemblages where this is not the case because a high percentage of species have warmer temperatures available within $1000 \mathrm{~km}$ of their range edge, assemblage exposure is still projected to occur abruptly (Extended Data Fig. 5), suggesting that other processes, such as ecological interactions ${ }^{24}$ or evolutionary conservatism in fundamental niches ${ }^{25,26}$, lead to similarity in realised niche limits ${ }^{16,27}$ and thus abruptness in the timing of exposure.

The synchronicity of species exposure within assemblages means the timing of assemblage-level exposure events is well-described by the median of species exposure times at a site (Extended Data Fig. 6). Under RCP8.5, the global mean year of assemblagelevel exposure is 2074 ( \pm 11 years s.d.), but there is considerable variation in the timing of exposure across assemblages (Fig. 2c). In some locations-such as the Caribbean and Coral Triangle-exposure is predicted to be already underway, with these hotspots of exposure expanding in spatial extent over time (Fig. 2c, Extended Data Fig. 7). By 2050, exposure spreads beyond ocean ecosystems to iconic terrestrial ecosystems, such as the Amazon and Congolese rainforests (Fig. 2c, Extended Data Fig. 7). Notably, the timing of these assemblage-level exposure events is not well predicted by the timing of local climate emergence (Spearman's $\rho=0.29$; Extended Data Fig. 5). And the timing of abrupt exposure events lags behind local climate emergence by 42 years (mean \pm 12 years s.d.), indicating the potential time-lag between climate change and ensuing biotic responses.

We find that the appearance of a gradual increase in risk to biodiversity globally can result from summarising across local assemblages that differ in their projected timing of abrupt exposure (Figs. 3c-d, Extended Data Fig. 8). Although these global summaries mask the abrupt nature of exposure within local assemblages, they can highlight the importance of increased mitigation efforts in reducing and delaying the onset of unprecedented climate conditions. Compared to RCP8.5, achieving RCP2.6 delays exposure for the most at risk 
species by circa six decades in the oceans (mean $=58$ years, range $=46-65$ years, Fig. $3 \mathrm{c}$ ) and on land (mean $=58$ years, range $=49-67$ years, Fig. $3 \mathrm{~d}$ ), buying valuable time for species and ecosystems, and human societies that depend upon them, to adapt to a warming climate.

\section{The risk of abrupt exposure events}

The abruptness of horizon profiles is positively correlated with the magnitude of exposure (Spearman's $\rho=0.58$; Extended Data Fig. 6), indicating a form of double jeopardy, whereby exposure events involving larger fractions of species are projected to occur more abruptly. This near-simultaneous exposure among multiple species could have sudden and devastating effects on local biodiversity and ecosystem services. Catastrophic, multispecies coral die-offs caused by a record-breaking marine heatwave in 2016 are one recent example ${ }^{6}$. Although it remains uncertain where "safe limits" of species loss for maintaining ecosystem function are located, meta-analyses suggest a $20 \%$ decline in species diversity as one possible threshold 28,29 . We therefore defined assemblages at risk of abrupt ecological disruption as those where at least $20 \%$ of species are projected to undergo exposure to unprecedented temperatures within the same decade. Holding warming to $<2^{\circ} \mathrm{C}$ above preindustrial levels limits such abrupt assemblage exposure events to $<2 \%$ of assemblages (Fig. 3e). However, beyond $2^{\circ} \mathrm{C}$ warming the area projected to undergo abrupt assemblage exposure expands rapidly, encompassing $15 \%$ of assemblages globally for $4^{\circ} \mathrm{C}$ warming. Furthermore, the increase in abrupt exposure does not differ markedly for assemblages afforded high habitat protection ( $\geq 20 \%$ protected area coverage of a grid cell), indicating that current protected areas are equally at risk from abrupt exposure (Fig. 3e).

The risk of abrupt exposure events differs across assemblages globally, with variability across individual climate projections increasing the total area at risk compared to median projections. For instance, even under RCP2.6 $\left(1.75^{\circ} \mathrm{C}\right.$ mean warming), $9 \%$ of assemblages are at some risk of abrupt exposure (Fig. 4a), increasing to $35 \%$ of assemblages under RCP8.5 $\left(4.4^{\circ} \mathrm{C}\right.$ mean warming; Fig. $\left.4 \mathrm{~b}\right)$. The risk of abrupt assemblage exposure events is positively correlated with species richness (RCP8.5, Spearman's $\rho=0.29$ (land) and 0.56 (ocean)), highlighting the increased risk of sudden ecological disruption in the world's most biodiverse ecosystems. Moreover, the risk of disruption of ecological function may be underestimated in this analysis because even if particular functional groups (e.g., habitat forming corals) suffer high levels of exposure, this may not be evident at the scale of entire assemblages if other groups are relatively less affected. When abrupt assemblage exposure events are instead defined at the level of major taxonomic groups, the area at risk expands further, encompassing 49\% of species assemblages under RCP8.5 (Fig. 4c, Extended Data Fig. 9). Our approach estimates how much of an assemblage's original biodiversity is exposed to potentially dangerous climate conditions over time ${ }^{28}$. We do not consider the potential for immigration of species from elsewhere to offset local biodiversity losses, but abrupt assemblage-wide exposure is likely to precipitate substantial ecological disruption regardless of the rate at which new species arrive. Furthermore, in tropical lowlands and oceans, where projected exposure is greatest, and warmer adapted species are lacking, net declines in local biodiversity are expected ${ }^{21}$.

\section{Crossing the biodiversity climate horizon}


217 While the horizon profile describes the accumulating number of co-occurring species 218 exposed to conditions beyond their realised niche limits, this need not equate with a profile of local extinction. Species may have wider fundamental than realised niche limits 13,30 , may avoid exposure in microclimatic refugia (but see Extended Data Fig. 10) or via behavioural thermoregulation ${ }^{17,31}$, or may evolve to tolerate novel conditions ${ }^{10}$. In these cases, the timing of abrupt assemblage exposure events could be considered an 'ignorance horizon', marking the time beyond which local extinctions are not inevitable but evidence for the ability of species to persist in the wild is largely absent ${ }^{13}$. Thus, at the very least, our results show that within 30 years, continued high emissions will drive a sudden shift across many ecological assemblages to climate conditions under which we have almost no knowledge of the ability of their constituent species to survive. Furthermore, we caution that the timing and magnitude of this exposure may occur earlier and be larger than we anticipate, because our analysis does not consider changes in extreme events 9 , effects of warming on local habitat (e.g. melting sea ice), covariation between climate variables ${ }^{32}$, or that populations may be locally adapted ${ }^{33}$.

To the extent that species realised historical thermal limits do reflect fundamental limits to persistence then the occurrence of abrupt exposure events marks the crossing of an 'ecological horizon' beyond which catastrophic and coordinated species losses are expected. These abrupt events-projected to spread from ocean (e.g., coral reef) to land (e.g., rainforest) ecosystems by 2050 under high emissions-risk sudden disruption to ecosystems and their capacity to maintain current levels of biodiversity and functioning. Evidence from lab and field-based studies indicates this is a credible risk, particularly for tropical terrestrial ectotherms and for marine organisms for which projected abruptness is most pronounced and for which realised geographic range boundaries most closely match thermal tolerance limits $16,18,30,34$. Indeed, warming over recent decades has already been associated with drastic population declines and local extinctions ${ }^{6,35,36}$, even amongst endotherms which are widely assumed to be less sensitive to warming but may be particularly vulnerable to climate driven disruption of trophic interactions ${ }^{37,38}$. For those ecosystems where exposure is projected within the next few decades, the capacity for species to adapt would appear limited. A priority for future research is to refine estimates of the timing and consequences of exposure, including where factors other than temperature may more strongly constrain species ranges, and where the emergence of novel climates has closest analogues deep in Earth's history ${ }^{39}$.

Considering the temporal dynamics of biodiversity exposure to climate change provides an early warning system of the potential for abrupt ecological disruption. Averting, or at least delaying, the crossing of this ecological horizon is possible for most assemblages, and requires massive and rapid reductions in greenhouse gas emissions. Elsewhere, our results highlight the urgency of targeted management responses, including establishing monitoring sites in exposed regions, new protected areas in refugia, and the potential for assisted migration and adaptation. 


\section{References}

1. Urban, M. C. Climate change. Accelerating extinction risk from climate change. Science 348, 571-573 (2015).

2. Warren, R., Price, J., Graham, E., Forstenhaeusler, N. \& VanDerWal, J. The projected effect on insects, vertebrates, and plants of limiting global warming to $1.5^{\circ} \mathrm{C}$ rather than $2^{\circ} \mathrm{C}$. Science 360, 791-795 (2018).

3. Newbold, T. Future effects of climate and land-use change on terrestrial vertebrate community diversity under different scenarios. Proc. Biol. Sci. 285, (2018).

4. Weber, C. et al. Mitigation scenarios must cater to new users. Nat. Clim. Chang. 8, 845-848 (2018).

5. Wernberg, T. et al. Climate-driven regime shift of a temperate marine ecosystem. Science 353, 169-172 (2016).

6. Hughes, T. P. et al. Global warming transforms coral reef assemblages. Nature 556, 492-496 (2018).

7. Barnosky, A. D. et al. Approaching a state shift in Earth's biosphere. Nature 486, 52-58 (2012).

8. Scheffer, M. et al. Early-warning signals for critical transitions. Nature 461, 53-59 (2009).

9. Harris, R. M. B. et al. Biological responses to the press and pulse of climate trends and extreme events. Nature Climate Change vol. 8 579-587 (2018).

10. Bay, R. A., Rose, N. H., Logan, C. A. \& Palumbi, S. R. Genomic models predict successful coral adaptation if future ocean warming rates are reduced. Sci Adv 3, e1701413 (2017).

11. Chevin, L.-M., Lande, R. \& Mace, G. M. Adaptation, Plasticity, and Extinction in a Changing Environment: Towards a Predictive Theory. PLoS Biol. 8, e1000357 (2010).

12. Colwell, R. K. \& Rangel, T. F. Hutchinson's duality: The once and future niche. Proceedings of the National Academy of Sciences 106, 19651-19658 (2009).

13. Feeley, K. J. \& Silman, M. R. Biotic attrition from tropical forests correcting for truncated temperature niches. Glob. Chang. Biol. 16, 1830-1836 (2009).

14. IUCN. The IUCN Red List of Threatened Species. Version 2017.

15. van Vuuren, D. P. et al. The representative concentration pathways: an overview. Clim. Change 109, 5-31 (2011).

16. Stuart-Smith, R. D., Edgar, G. J. \& Bates, A. E. Thermal limits to the geographic distributions of shallow-water marine species. Nat Ecol Evol 1, 1846-1852 (2017).

17. Sunday, J. M. et al. Thermal-safety margins and the necessity of thermoregulatory behavior across latitude and elevation. Proc. Natl. Acad. Sci. U. S. A. 111, 5610-5615 (2014).

18. Dillon, M. E., Wang, G. \& Huey, R. B. Global metabolic impacts of recent climate warming. Nature 467, 704-706 (2010).

19. Hawkins, E. \& Sutton, R. Time of emergence of climate signals. Geophys. Res. Lett. 39, (2012).

20. Mora, C. et al. The projected timing of climate departure from recent variability. Nature $\mathbf{5 0 2}$, 183-187 (2013).

21. Colwell, R. K., Brehm, G., Cardelús, C. L., Gilman, A. C. \& Longino, J. T. Global warming, elevational range shifts, and lowland biotic attrition in the wet tropics. Science 322, 258261 (2008).

22. IPCC, 2013: Climate Change 2013: The Physical Science Basis. Contribution of Working Group I to the Fifth Assessment Report of the Intergovernmental Panel on Climate Change. (Cambridge University Press).

23. Williams, J. W., Jackson, S. T. \& Kutzbach, J. E. Projected distributions of novel and disappearing climates by 2100 AD. Proc. Natl. Acad. Sci. U. S. A. 104, 5738-5742 (2007). 
24. Liautaud, K., van Nes, E. H., Barbier, M., Scheffer, M. \& Loreau, M. Superorganisms or loose collections of species? A unifying theory of community patterns along environmental gradients. Ecol. Lett. 22, 1243-1252 (2019).

25. Araújo, M. B. et al. Heat freezes niche evolution. Ecol. Lett. 16, 1206-1219 (2013).

26. Crisp, M. D. et al. Phylogenetic biome conservatism on a global scale. Nature 458, 754-756 (2009).

27. White, A. E., Dey, K. K., Mohan, D., Stephens, M. \& Price, T. D. Regional influences on community structure across the tropical-temperate divide. Nat. Commun. 10, 2646 (2019).

28. Newbold, T. et al. Has land use pushed terrestrial biodiversity beyond the planetary boundary? A global assessment. Science 353, 288-291 (2016).

29. Hooper, D. U. et al. A global synthesis reveals biodiversity loss as a major driver of ecosystem change. Nature 486, 105-108 (2012).

30. Sunday, J. M., Bates, A. E. \& Dulvy, N. K. Thermal tolerance and the global redistribution of animals. Nature Climate Change vol. 2 686-690 (2012).

31. Pinsky, M. L., Eikeset, A. M., McCauley, D. J., Payne, J. L. \& Sunday, J. M. Greater vulnerability to warming of marine versus terrestrial ectotherms. Nature 569, 108-111 (2019).

32. Mahony, C. R. \& Cannon, A. J. Wetter summers can intensify departures from natural variability in a warming climate. Nature Communications vol. 9 (2018).

33. Valladares, F. et al. The effects of phenotypic plasticity and local adaptation on forecasts of species range shifts under climate change. Ecol. Lett. 17, 1351-1364 (2014).

34. Deutsch, C. A. et al. Impacts of climate warming on terrestrial ectotherms across latitude. Proc. Natl. Acad. Sci. U. S. A. 105, 6668-6672 (2008).

35. Sinervo, B. et al. Erosion of lizard diversity by climate change and altered thermal niches. Science 328, 894-899 (2010).

36. Soroye, P., Newbold, T. \& Kerr, J. Climate change contributes to widespread declines among bumble bees across continents. Science 367, 685-688 (2020).

37. Lister, B. C. \& Garcia, A. Climate-driven declines in arthropod abundance restructure a rainforest food web. Proc. Natl. Acad. Sci. U. S. A. (2018) doi:10.1073/pnas.1722477115.

38. Spooner, F. E. B., Pearson, R. G. \& Freeman, R. Rapid warming is associated with population decline among terrestrial birds and mammals globally. Glob. Chang. Biol. 24, 4521-4531 (2018).

39. Burke, K. D. et al. Pliocene and Eocene provide best analogs for near-future climates. Proc. Natl. Acad. Sci. U. S. A. 115, 13288-13293 (2018). 

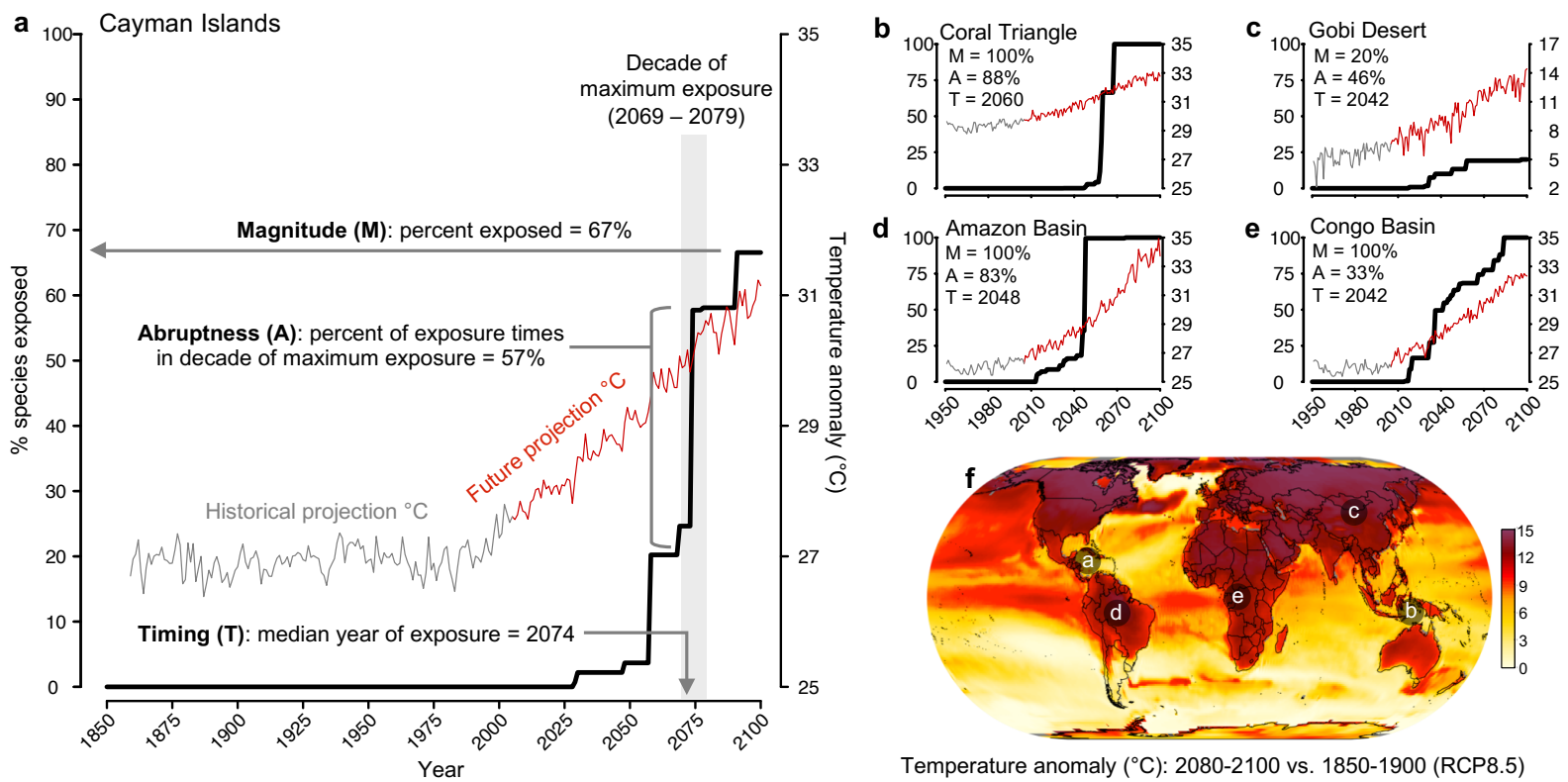

e ${ }_{100}$ Congo Basin
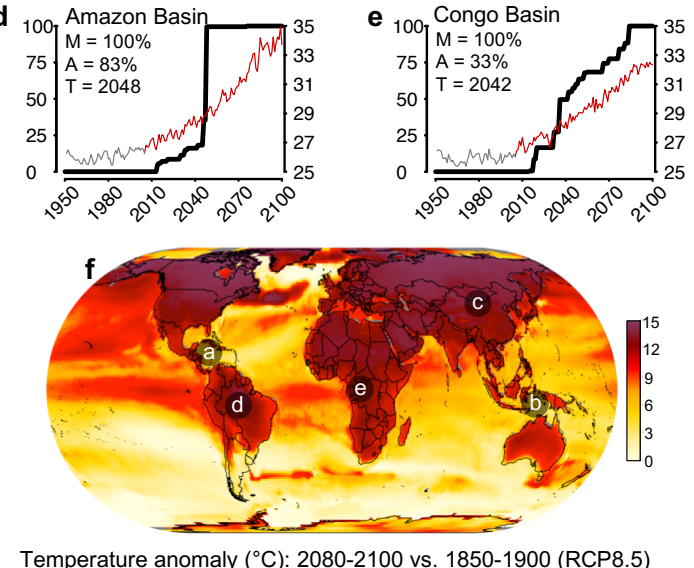

Temperature anomaly $\left({ }^{\circ} \mathrm{C}\right): 2080-2100$ vs. $1850-1900$ (RCP8.5)

Figure 1. Biodiversity climate horizon profiles. Profiles (solid black lines) indicate the cumulative percent of species in an assemblage exposed to future temperatures (red line) beyond their realised thermal niche over time. Iconic ecosystems provide examples of different profile shapes; a, Cayman Islands, b, Coral Triangle, c, Gobi Desert, d, Amazon Basin and $\mathbf{e}$, Congo Basin (see $\mathbf{f}$, map of temperature anomalies for locations). Horizon profiles and temperature trends are shown for a single run of the Hadley Centre Global Environmental Model (HadGEM2) under a high greenhouse gas emissions scenario (RCP8.5). Timing, magnitude and abruptness define differences among profiles. Grey line shows historical temperature projections at a site. 

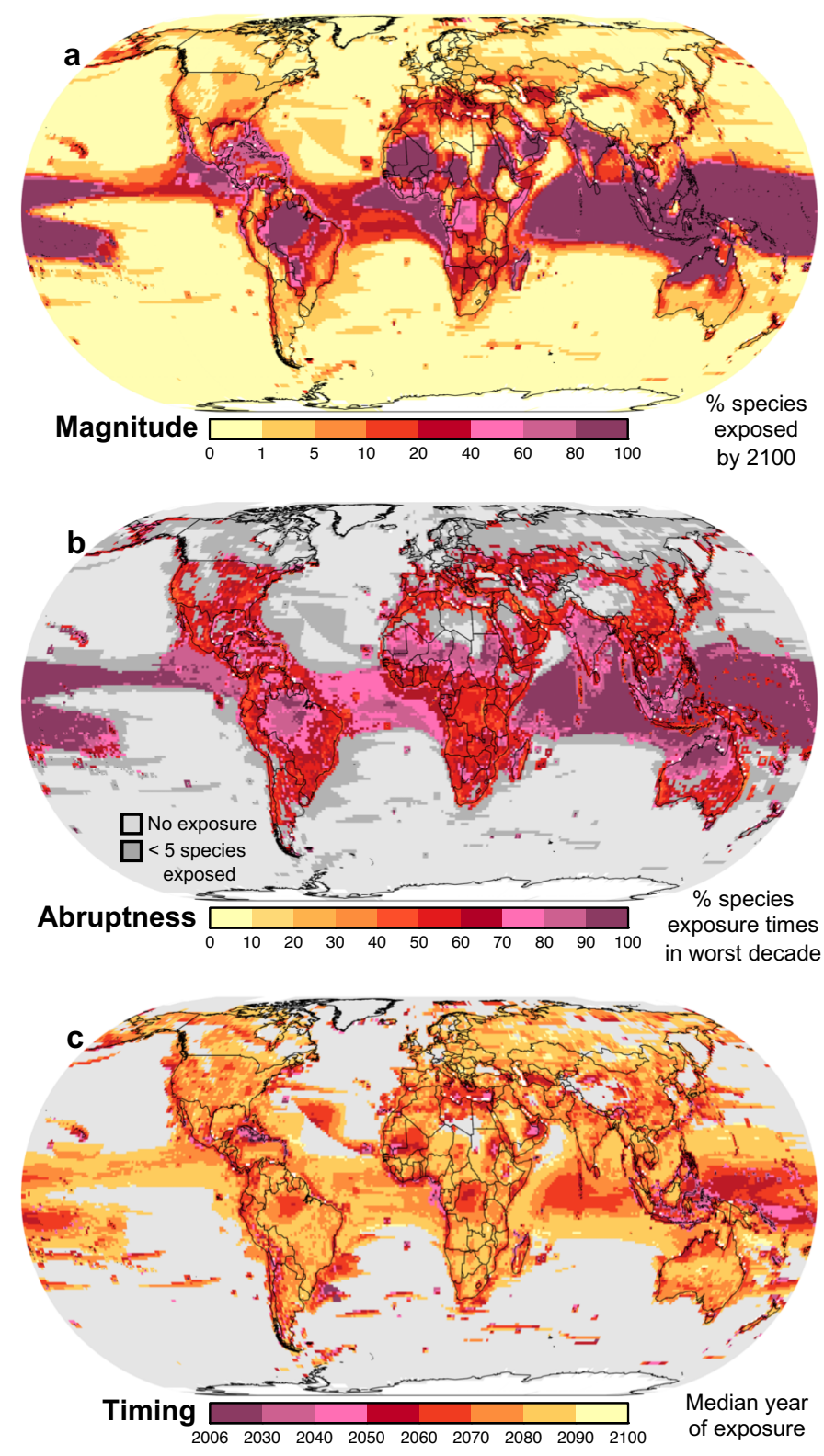

353 Figure 2. Global variation in the magnitude, abruptness and timing of horizon

354 profiles. a, magnitude of exposure indicating the percent of species in $100 \mathrm{~km}$ resolution 355 grid cells (i.e., assemblages) exposed to unprecedented temperature (i.e., beyond each 356 species' realised niche) by 2100 . b, abruptness quantified as the percent of species 357 exposure times occurring within the decade of maximum exposure for each assemblage. c, 358 timing quantified as the median year of local species exposure conditional on being 359 exposed by 2100 , the end of the simulation. Maps show the median value across 22 climate 360 models under RCP8.5 (see Extended Data Fig. 1 for RCPs 2.6 and 4.5). 

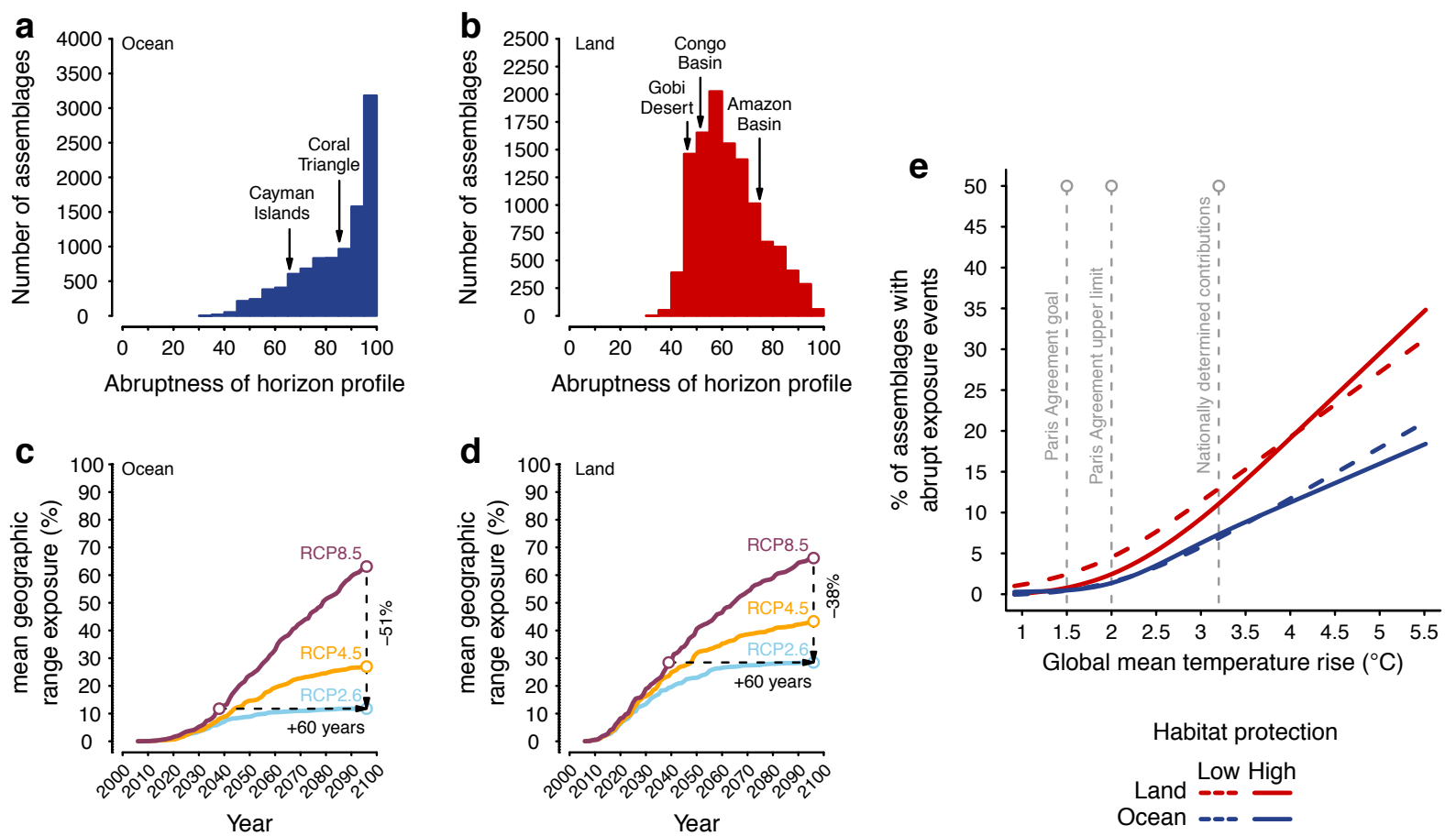

Figure 3. Abruptness of horizon profiles locally versus globally, and accelerating risk with global warming. The distribution in the projected abruptness of species exposure to unprecedented temperatures within marine and terrestrial assemblages (a,b). Selected assemblages from Fig. 1 are highlighted. Abruptness is quantified as the percent of species exposure times occurring within the decade of maximum exposure, with results showing the median across climate models under RCP8.5. Global horizon profiles for oceans and land (c,d) showing more gradual accumulation of species exposure to unprecedented temperatures. Dashed lines show how lowering emissions from RCP8.5 to RCP2.6 both reduces the median magnitude of exposure across climate models and substantially delays the timing of exposure, buying 60 years for species and conservation plans to adapt to a warming climate (see Extended Data Fig. 8 for individual climate models). e, the percent of species assemblages projected to experience high magnitude and abrupt assemblage exposure ( $>20 \%$ of species exposed in a single decade) as a function of global warming. Curves are fitted from model runs $(n=66)$ across RCP2.6, RCP4.5 and RCP8.5. 

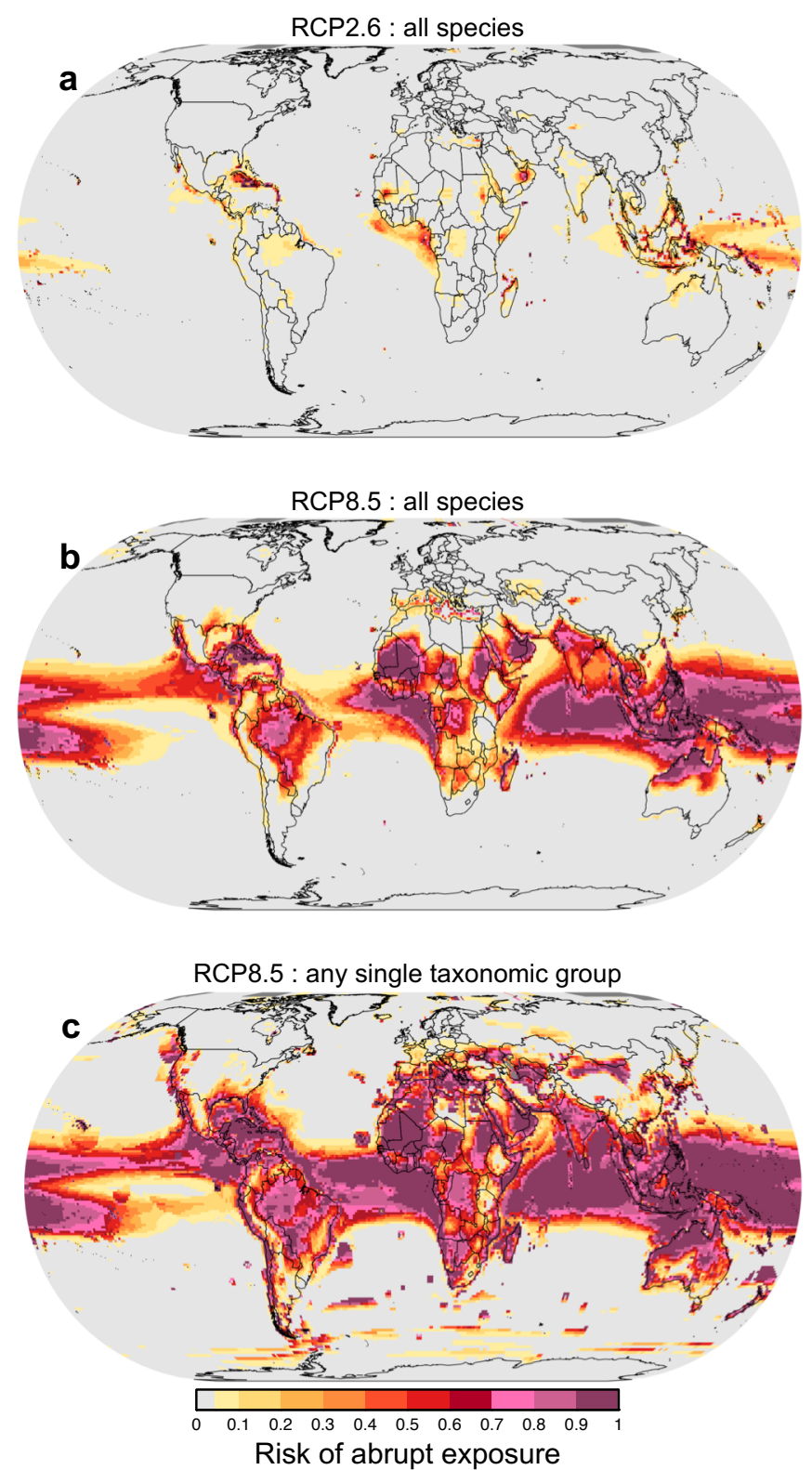

377 Figure 4. The risk of high magnitude, abrupt assemblage exposure events. Risk is calculated as the proportion of 22 climate models where an abrupt exposure event is projected to occur before 2100. Assemblages avoiding abrupt exposure events across all 22 models are in grey. In a-b, abrupt exposure events are defined as $>20 \%$ of all species in an assemblage exposed in a single decade. In c, abrupt exposure events are defined when any single organism group (e.g., amphibians) within an assemblage exhibits the exposure of $>20 \%$ of its constituent species in a single decade, highlighting the widespread risk of abrupt

384 ecological disruption. 
388 Biodiversity data. We used expert verified range maps for 30,652 species from the International Union for Conservation of Nature (IUCN) ${ }^{14}$ and BirdLife International ${ }^{40}$, including; birds, mammals, reptiles, amphibians, marine fish, benthic marine invertebrates, and habitat forming corals and seagrasses (Supplementary Table 1). To further increase coverage of open ocean assemblages, our sample includes additional data on $\mathrm{krill}^{41}$ and cephalopods $^{42}$, reflecting the availability of expert range maps for oceanic species ${ }^{43}$. We used only breeding ranges for terrestrial species. We excluded marine species restricted to depths greater than 200 meters (the lower limit of the epipelagic zone), as these species are less likely to respond to changes in sea surface temperature. Range maps were converted to $100 \mathrm{~km}$ resolution equal-area grid cells, the finest resolution justifiable for these data globally without incurring false presences ${ }^{44,45}$. Expert range maps provide comprehensive information on species' global geographic distributions ${ }^{46}$, but our results should be interpreted in the context of known data limitations. For some groups, species coverage is incomplete and biased towards commercial species (e.g. cephalopods), while others have been comprehensively assessed for only a subset of clades (e.g. fish) and the species included in our study thus represent a non-random subset of global biodiversity (Supplementary Table 1). For instance, insects and plants may on average be more at risk of geographic range loss due to climate change than terrestrial vertebrates ${ }^{2}$, but we did not assess exposure for these groups because range maps (expert or otherwise) are not available globally. As such, both very short-lived and long-lived terrestrial taxa may be underrepresented in our sample. Furthermore, while many IUCN range maps consider occurrence data from historical records, others may underestimate climate niche limits where longer-term historical records are unavailable and recent geographic range contractions have occurred in part due to reasons other than climate change ${ }^{47}$.

Data on marine and terrestrial protected areas were downloaded from the World Database on Protected Areas (http://protectedplanet.net/; accessed 21st March 2018). The maps, originally in polygon format, were re-sampled to a $1 \mathrm{~km}$ resolution prior to further analysis. We considered $100 \mathrm{~km}$ resolution grid cells highly protected if at least $20 \%$ of the grid cell was inside protected areas.

Climate model data. We used temperature and precipitation projections from 22 General Circulation and Earth System Models developed for CMIP5 (Supplementary Table 2). For each model, we downloaded a single projection for mean monthly precipitation ( $\mathrm{mm}$ ), near-surface temperature $(\mathrm{K})$ and sea surface temperature $(\mathrm{K})$ for the historical run (1850-2005), as well as RCP2.6, RCP4.5 and RCP8.5 scenarios for the years 2006-2100 or 2006-2300, when available. Model output was downloaded from https://esgfnode.llnl.gov/projects/esgf-llnl/ (accessed 5th June 2017). In our main analysis, we focus on the dynamics of exposure according to mean annual temperature (MAT), calculated by averaging monthly values. However, we also repeated our analysis using the temperature of the hottest month, hereafter 'maximum monthly temperature' [MMT], and for terrestrial assemblages, total annual precipitation $(\mathrm{mm})$, calculated by summing precipitation values across months (see Supplementary Information). Note that the identity of the hottest month can vary both across sites and between years within a site. Given that CMIP5 models use different spatial grids, and to match the resolution of species geographic range data, 
climate model data were regridded to a $100-\mathrm{km}$ resolution grid using an area-weighted mean interpolation. Climate data interpolation was done in $\mathrm{CDO}^{48}$ and $\mathrm{R}^{49}$.

We calculated species exposure times for each assemblage using individual climate simulations, as opposed to ensembles or multi-model averages, because individual simulation runs include variance in climatic time series due to internal climate variability such as the timing of El Niño-Southern Oscillation events ${ }^{22,50}$. This internal variability is a key component of the uncertainty in the timing of exposure, and is smoothed out if using multi-model averages as input into the analysis. By calculating species exposure events using individual model simulation runs and then summarising across models, we capture the uncertainty in the timing of exposure due to both internal climate variability and climate model uncertainty (i.e., uncertainty about climate physics across models), in line with 'time of emergence' analyses from climate science ${ }^{19}$. Throughout, we report multimodel medians in each of our summary metrics.

Defining species realised niche limits. Species experience variability in climatic conditions across both space and time but this temporal variability is ignored when using time-averaged climate conditions (e.g., Worldclim data ${ }^{51}$ ) to estimate species realised niches. To address this, we estimated species realised niche limits using the climate projections from the historical run of each climate model (1850-2005), which includes the influence on climate of observed changes in radiative forcing due to natural factors such as volcanic eruptions, as well as anthropogenic emissions and land use changes ${ }^{52}$. Thus, in the case of MAT, we calculated the maximum MAT experienced across the species geographic range over both space and time (Tmax ${ }_{\mathrm{MAT}}$, see Supplementary Information). To prevent estimates of TmaxмAT being inflated by either extreme outliers in the temperature time series or from the overestimation of species ranges ${ }^{44}$, we excluded outlier temperature values within each grid cell, defined as those more than three standard deviations from the mean. Once we had selected the maximum temperature for each cell, we excluded outlier temperature values across each species range, defined as those more than three standard deviations above the mean range value. The Tmaxмат value for each species was then set as the maximum of the remaining values (Supplementary Fig. 1). We used an identical

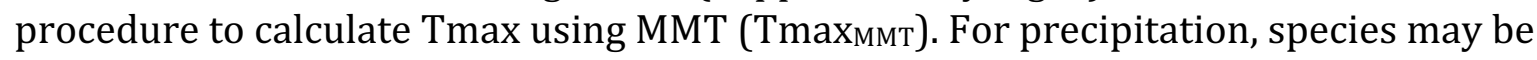
exposed to either drying or wetting conditions and so we calculated both the minimum (Pmin) and maximum (Pmax) precipitation value experienced by each species across its geographic range (see Supplementary Information).

Estimating species exposure times. Within each terrestrial $(n=18560)$ and marine $(n=$ 37333) assemblage (i.e. $100 \mathrm{~km}$ grid cell containing any terrestrial or marine species respectively), we defined the time of local species exposure to unprecedented temperature (i.e. the 'climate horizon') to be the year after which the MAT (or MMT) of the cell is

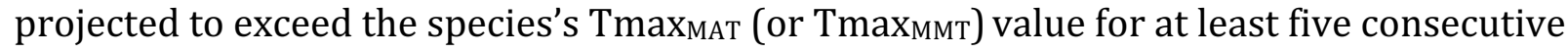
years. We note that using a higher number of consecutive years ( $n=20$ years) had little effect on the magnitude, timing or abruptness of exposure (Supplementary Figs. 2 and 3).

For precipitation, we calculated the time of local species exposure as the year after which the precipitation of the cell is projected to be either above or below the species' Pmax and Pmin values respectively for at least five consecutive years. Annual precipitation values are bounded at zero and this could potentially lead to exposure being 
underestimated for locations projected to have historically received zero precipitation. To address this, we additionally defined exposure when annual precipitation fell below $15 \mathrm{~mm}$ for at least five consecutive years. Due to the generally weaker trends and high variability in historical and future projected precipitation, we found that few species were exposed to unprecedented precipitation regardless of how exposure was defined (Extended Data Fig. 1). To show the importance of increasing temperatures as the primary driver of exposure, we compared patterns of exposure from MAT alone to those from MAT and precipitation combined, recording the earliest local exposure time of either MAT or precipitation for a species in an assemblage when it was exposed to both variables (Extended Data Fig. 2g-i, see Supplementary Information).

We note that by using range-wide estimates of species niche limits, we may underestimate both the magnitude and immediacy of exposure if populations are locally adapted $^{33}$. Unfortunately, information on the scale and strength of local adaptation is not generally available across species. Equally, our analysis does not attempt to model adaptive evolution, which may enable species to shift or expand their climatic niche limits over time. Nevertheless, our estimates of the timing of local exposure to unprecedented conditions may be relevant for understanding the potential for evolution to rescue populations from changing climates ${ }^{10,11 .}$

Horizon profiles. Once species exposure times had been calculated for an assemblage we constructed a horizon profile indicating the cumulative percentage of species locally exposed to conditions beyond their realised niche limits. We used the following metrics to summarise the temporal dynamics of biodiversity exposure. First, we calculated the magnitude of exposure as the percent of species in the assemblage exposed over the course of the $21^{\text {st }}$ century. Second the abruptness of exposure for an assemblage was calculated as the percent of all exposure times that occur in the decade of maximum exposure. We identified the decade of maximum exposure using a moving window of ten years. We also calculated an alternative metric of abruptness based on the Shannon-entropy index ${ }^{53}$, which quantifies the evenness in the distribution of exposure times across all years of the horizon profile (Extended Data Fig. 4). In contrast to our original abruptness metric, lower values of the Shannon-entropy index indicate a more abrupt profile. We therefore rescaled the Shannon-entropy index by the maximum possible entropy value per assemblage, subtracted these values from 1 and then multiplied by 100 to give an index ranging between 0 and 100, where a value of 100 indicates that all exposure times occur in a single decade and a value of 0 corresponds to an equitable distribution of exposure times across years. Abruptness was only calculated for assemblages where five or more species were exposed to avoid idiosyncrasies due to small sample sizes. Third, the timing of exposure for each assemblage was calculated as the median of the times of local species exposure events. Species not exposed before the end of the $21^{\text {st }}$ century were excluded from this calculation. We repeated our analysis using alternative metrics of timing, including the mean year of exposure and the mid-point of the decade of maximum exposure, obtaining very similar results (Extended Data Fig. 6). For each of these exposure metrics we report the median value across the 22 climate models for a given climate scenario, and quantify uncertainty as the standard deviation (Extended Data Fig. 3). The greatest uncertainty in projected impacts involves the magnitude of exposure along the boundaries of the tropics. This arises because of variation among models in the magnitude of warming which alters 
the spatial extent of regions exposed to unprecedented temperatures. In contrast, variation among models in the timing and abruptness of exposure is relatively small and does not exhibit any clear spatial structure.

We compared the median timing of species exposure within assemblages to the timing of local climate emergence, defined as the year after which future local temperatures are projected to exceed the maximum historical (1850-2005) conditions at a site $^{19,20}$. Timing of emergence was calculated using an identical procedure to the timing of exposure, excluding outlying values from the time series when quantifying the maximum historical temperature at a site and only considering emergence when temperatures exceed the historical maximum for at least five consecutive years. The time of local climate emergence at a site is therefore identical to the time of local exposure for a species occupying a single grid cell. In the absence of perfect adaptation to local climates, a time-lag is therefore expected between local climate emergence and the median timing of exposure, because species typically persist under a broader range of conditions than is present in any single site.

Spatial scale. We modelled species realised niche limits using climate projections at $100 \mathrm{~km}$ grain size, matching the resolution of expert geographic range maps ${ }^{44,45}$. However, individual grid cells at this resolution may contain (potentially substantial) spatial climatic heterogeneity, thus potentially underestimating variability in species niche limits and potentially overestimating the abruptness of assemblage exposure dynamics. To investigate this possibility, we tested whether the abruptness of horizon profiles across terrestrial assemblages is related to the range in mean annual temperatures within each grid cell, using spatially interpolated weather data for the period 1970-2000 available at $1 \mathrm{~km}$ resolution ${ }^{51}$. We found that abruptness is negatively correlated with the spatial heterogeneity in temperature within a cell (Spearman's $\rho=-0.29$ ), so that assemblages with higher spatial heterogeneity in temperatures (e.g. tropical mountains), exhibit more gradual exposure profiles than those with low heterogeneity in temperatures (e.g. tropical lowlands) (Extended Data Fig. 10). This result has two important implications. First, it suggests that despite the relatively coarse grain size, our analysis still identifies those assemblages where variation in realised niche limits among species is expected to be greatest (i.e. grid cells containing substantial spatial climatic heterogeneity) as having the most gradual exposure profiles. Second, it suggests that while incorporating finer-scale climate data may further reduce the lowest abruptness values estimated across assemblages (i.e. making relatively gradual horizon profiles more gradual), it is unlikely to alter the key conclusion that assemblage exposure to climate warming occurs abruptly, because the most abrupt horizon profiles occur in assemblages where there is little finescale climatic heterogeneity (Extended Data Fig. 10). These results support the robustness of our overall conclusions regarding the dynamics of exposure, but it is clear that increasing the spatial resolution at which species niche limits and assemblages are defined would enable a more precise quantification of the timing of species exposure to changing climates and should be a priority for future research.

Horizon profiles can be calculated either for a single assemblage or for a set of assemblages combined, such as a biome or the entire globe. In addition to examining the dynamics within assemblages, we generated global horizon profiles, describing the total cumulative exposure of all populations (i.e. species by site combinations) across marine 
603

604

605

606

607

608

609

610

611

612

613

614

615

616

and terrestrial assemblages (Fig. 3c-d). To avoid exposure dynamics being driven by the small number of species with the largest geographic ranges, we weighted each species by the inverse of its geographic range size. This range-size weighted exposure profile ensures that each species contributes equally to exposure dynamics and is mathematically equivalent to calculating the mean \% geographic range exposure across species. Unweighted global horizon profiles show qualitatively similar patterns (Extended Data Fig. 8).

Risk of abrupt exposure events. We identified those assemblages projected to undergo abrupt and high magnitude exposure events, defined as at least $20 \%$ of resident species exposed within a single decade before the end of the $21^{\text {st }}$ century. Across the set of 66 climate model runs from the three RCP scenarios, we fit a generalised additive model (GAM) to estimate the \% of assemblages projected to undergo abrupt exposure events as a function of mean global warming at the end of the century (2080-2100) relative to preindustrial conditions (1850-1900). We fit separate models for sites with either low or high (i.e. $>20 \%$ in protected areas) levels of habitat protection. We forced the regression through the origin, thus assuming no abrupt exposure events would occur if temperatures remained stable at pre-industrial conditions. Because the identity of assemblages projected to undergo abrupt exposure events may vary across model runs, the actual area at risk of abrupt exposure may be substantially greater than expected under any single climate simulation. For each assemblage, we therefore calculated the probability of an abrupt exposure event across the 22 climate models within each emissions scenario. We did this for assemblages consisting of all species, as well as for each organism group separately.

\section{References}

40. Birdlife International. Bird species distribution maps of the world. Version 2.0. Cambridge/Arlington, TX: BirdLife International/NatureServe (2012).

41. Brinton, E., Ohman, M. D., Townsend, A. W., Knight, M. D. \& Bridgeman, A. L. Euphausiids of the World Ocean. (Springer, 2000).

42. Jereb, P. \& Roper, C. F. E. Cephalopods of the World: An Annotated and Illustrated Catalogue of Cephalopod Species Known to Date. (2005).

43. Tittensor, D. P. et al. Global patterns and predictors of marine biodiversity across taxa. Nature 466, 1098-1101 (2010).

44. Hurlbert, A. H. \& Jetz, W. Species richness, hotspots, and the scale dependence of range maps in ecology and conservation. Proc. Natl. Acad. Sci. U. S. A. 104, 13384-13389 (2007).

45. Jetz, W., Sekercioglu, C. H. \& Watson, J. E. M. Ecological correlates and conservation implications of overestimating species geographic ranges. Conserv. Biol. 22, 110-119 (2008).

46. Meyer, C., Kreft, H., Guralnick, R. \& Jetz, W. Global priorities for an effective information basis of biodiversity distributions. Nat. Commun. 6, 8221 (2015).

47. Faurby, S. \& Araújo, M. B. Anthropogenic range contractions bias species climate change forecasts. Nature Climate Change vol. 8 252-256 (2018).

48. Schulzweida, U. CDO User Guide (Version 1.9.6). URL http://doi.org/10.5281/zenodo.2558193. (2019, February 6).

49. R Core Team. R: A language and environment for statistical computing. R Foundation for Statistical Computing, Vienna, Austria. URL http://www.R-project.org/. (2019). 
50. Kay, J. E. et al. The Community Earth System Model (CESM) Large Ensemble Project: A Community Resource for Studying Climate Change in the Presence of Internal Climate Variability. Bull. Am. Meteorol. Soc. 96, 1333-1349 (2015).

51. Hijmans, R. J., Cameron, S. E., Parra, J. L., Jones, P. G. \& Jarvis, A. Very high resolution interpolated climate surfaces for global land areas. Int. J. Climatol. 25, 1965-1978 (2005).

52. Taylor, K. E., Stouffer, R. J. \& Meehl, G. A. An Overview of CMIP5 and the Experiment Design. Bull. Am. Meteorol. Soc. 93, 485-498 (2012).

53. Shannon, C. E. A Mathematical Theory of Communication. Bell System Technical Journal vol. 27 623-656 (1948). 


\section{Acknowledgements}

628 We are grateful to Georgina Mace and Owen Petchey for their helpful comments on pre-

629 submission drafts of the manuscript. This study has been supported by the following

630 institutions and grants to A.L.P (The Royal Society, UK), C.H.T (National Socio-

631 Environmental Synthesis Center under funding received from the National Science

632 Foundation DBI-1639145 and the FLAIR Fellowship Programme: a partnership between

633 the African Academy of Sciences and the Royal Society funded by the UK Government's

634 Global Challenges Research Fund) and C.M (NSF Grants 1565046 and 1661510).

635

636

637

638

639

640

641

642

643

644

645

646

647

648

649

650

651

652

653

654

655

656

657

658

659

660

661

662

663

664

665

666

667

668

669

670

671

\section{Author Information}

\section{Affiliations}

African Climate and Development Initiative, University of Cape Town, South Africa.

Christopher H. Trisos

National Socio-Environmental Synthesis Center (SESYNC), 1 Park Place, Suite 300,

Annapolis, MD 21401, USA.

Christopher H. Trisos

Centre for Statistics in Ecology, the Environment, and Conservation, University of Cape

Town, South Africa

Christopher H. Trisos

Ecology and Evolutionary Biology, University of Connecticut, Storrs, CT 06269, USA

Cory Merow

Ecology and Evolutionary Biology, Yale University, New Haven, CT 06511, USA

Cory Merow

Centre for Biodiversity and Environment Research, Department of Genetics, Evolution and

Environment, University College London, Gower Street, London, WC1E 6BT, UK.

Alex L. Pigot

\section{Contributions}

A.L.P, C.H.T, C.M. conceived the study, processed the species and climate data, performed the analysis and wrote the manuscript. All authors read and approved the final manuscript.

\section{Competing interests}

The authors declare no competing financial interests.

\section{Correspondence}

Correspondence and requests for information should be addressed to Alex Pigot:

a.l.pigot@ucl.ac.uk

\section{Reprints and permissions}

Reprints and permissions information is available at www.nature.com/reprints.

\section{Data availability}

All datasets used here are publicly available. Expert verified range maps are available from https://www.iucnredlist.org/resources/spatial-data-download and 
672 http://datazone.birdlife.org/species/requestdis. Climate change projections for RCPs 8.5, 673 4.5, and 2.6 for the Coupled Model Intercomparison Project 5 (CMIP5) are available from 674 https://esgf-node.llnl.gov/search/cmip5/. Computer code used in the analysis is available 675 on request from the authors. Code and results data to make figures 2-4 is available at 676 figshare (10.6084/m9.figshare.11814633). 
677 Extended Data figure 1. Spatial distribution of the magnitude, abruptness and timing, of assemblage exposure for alternative climate variables. Results show the median value across 22 CMIP5 climate models for mean annual temperature, maximum monthly

680 temperature, and precipitation under RCP2.6, RCP4.5 and RCP8.5.

\section{Extended Data figure 2. Comparing the magnitude, timing and abruptness of}

682 assemblage exposure across alternative climate variables. a-f, patterns of exposure to unprecedented temperatures show both similarities and differences depending on whether temperature is quantified using mean annual temperature (MAT) or maximum monthly temperatures (MMT). More species are exposed and exposure occurs earlier for MAT compared to MMT, but spatial variation in the magnitude $(\mathbf{a}, \mathbf{d})$ and timing $(\mathbf{b}, \mathbf{e})$ of exposure are strongly correlated between temperature variables. Variation in the abruptness of assemblage exposure is less strongly correlated between MAT and MMT (c), but both variables confirm the abruptness of projected exposure (f). g-i, patterns of exposure to both MAT and precipitation combined are very similar to patterns of exposure to only MAT, highlighting the primacy of changes in temperature in driving exposure. Values are the median across 22 CMIP5 climate models under RCP8.5, with hotter colors indicating a higher density of points. In (a-c) and (g-i) points falling along the dashed 1:1 line indicate a perfect correspondence between metrics. The correlation between metrics (Spearman's $\rho$ ), and the mean difference in the timing of exposure (years), is shown.

Extended Data figure 3. Uncertainty (standard deviation, SD) in species local exposure metrics across 22 CMIP5 climate models under RCP8.5. Uncertainty in the magnitude of exposure is greatest around the boundaries of the tropics with little geographic variation in uncertainty in timing or abruptness.

700 Extended Data figure 4. Abruptness of horizon profiles. Density plots show the distribution of abruptness values for different CMIP5 climate models ( $n=22$, lines) and representative concentration pathways (RCPs) on land (red) and in the ocean (blue).

703

704

705 Histograms show the median abruptness across climate models under RCP8.5 for each organism group. Abruptness is calculated as the percent of exposure times occurring within the decadal window of maximum exposure (colors). Abruptness is also shown for an alternative metric based on the Shannon-entropy index (grey) with values scaled between 0 and 100 , indicating the most gradual and abrupt distribution of exposure times possible for a given assemblage respectively. Exposure is consistently abrupt across climate models, RCP scenarios, metrics and organism groups.

711

712

713

714

715

716

Extended Data figure 5. Predicting the timing, magnitude and abruptness of local species exposure. On land (left) and in the ocean (right) the (a-b) median timing of exposure is weakly correlated (Spearman's $\rho$ ) with the timing of local climate emergence. The magnitude of exposure (c-d) is weakly correlated with the magnitude of warming between the start (2000-2020) and the end (2090-2100) of the $21^{\text {st }}$ Century. The abruptness of exposure (\% of local species exposure times occurring in the decade of maximum exposure) is only partly correlated with (e-f) the maximum rate of warming (maximum difference in mean temperature between successive decades) or (g-h) the $\%$ of species with nowhere warmer within $1000 \mathrm{~km}$ of their range. Values are the median across 22 CMIP5 climate models under RCP8.5. Hotter colors indicating a higher density of points. 
Extended Data figure 6. The different dimensions of climate risk to species assemblages. a-c, Bivariate plots showing the strong correlation among alternative metrics for the timing of local assemblage exposure: the median year of local species exposure, the mean year of local species exposure and the mid-point of the decadal window of worst (i.e. maximum) local species exposure. d-f, Bivariate plots showing the weaker correlation between the magnitude, abruptness and timing of exposure across assemblages. Values are the median across 22 CMIP5 climate models under RCP8.5, with hotter colors indicating a higher density of points. In (a-c) points falling along the dashed 1:1 line indicate a perfect correspondence between metrics. The correlation between metrics (Spearman's $\rho$ ) is shown, as well as for (a-c) the mean difference in the timing of exposure (years).

Extended Data figure 7. Accumulation of exposure to unprecedented temperatures at decadal time snapshots from 2030 to 2100 . Light grey indicates zero local species exposure. Maps show the median across 22 CMIP5 climate models under RCP8.5, highlighting the immediate onset of exposure in the tropics that spreads to higher latitudes later in the century.

\section{Extended Data figure 8. The global biodiversity horizon profile. The cumulative} exposure to unprecedented temperatures of all local species populations (i.e. species X site aggregated across all sites) increases smoothly over time at the global scale. Global horizon profiles are shown when (a-b) species are weighted by the inverse of their geographic range size (equivalent to the mean \% geographic range exposed) or (d-f) are given equivalent weighting. In (d-f), dynamics are dominated by species with many local populations (i.e. large geographic ranges). Variability in exposure across 22 climate models (thin lines) is shown for each RCP scenario (median, thick line).

Extended Data figure 9. The global distribution in the risk of high-magnitude and abrupt assemblage exposure events under different representative concentration pathways. Maps show the probability of abrupt exposure calculated across 22 CMIP5 climate models. The risk of abrupt exposure was calculated based on all species in an assemblage (left column) and for each organism group separately (right column). Maps highlight the greater risk of abrupt exposure events under intermediate (RCP4.5), and especially high (RCP8.5) emission pathways, and when considering taxonomic groups separately

Extended Data figure 10. Abruptness of horizon profiles for terrestrial vertebrates in $100 \mathrm{~km}$ grid cells with low (red, $1^{\text {st }}$ quantile) or high (grey, $4^{\text {th }}$ quantile) spatial temperature heterogeneity. Abruptness is calculated as the $\%$ of species exposure times in the decade of maximum exposure. Temperature heterogeneity is the range in temperatures at $1 \mathrm{~km}$ resolution within each $100 \mathrm{~km}$ cell. Assemblages with abrupt exposure have lower temperature heterogeneity suggesting that quantifying species niches at finer spatial resolutions is unlikely to alter the abrupt nature of assemblage exposure dynamics. 